\title{
ASSIMETRIA FLUTUANTE: DADOS INICIAIS DE UMA TAXOCENOSE DE SABIÁS (PASSERIFORMES, TURDIDAE) OCORRENTES EM UM PARQUE URBANO NA REGIÃO CENTRO-SUL DO ESTADO DO PARANÁ, BRASIL
}

\author{
FLUCTUATING ASYMMETRY: EARLY DATA FROM A TAXOCENOSE OF \\ THRUSHES (PASSERIFORMES, TURDIDAE) IN AN URBAN PARK IN THE \\ MIID-SOUTH REGION OF THE STATE OF PARANÁ, BRAZIL
}

\author{
Huilquer Francisco Vogel ${ }^{1}$, Cláudio Henrique Zawadzki², Gustavo Sene Silva ${ }^{3}$, \\ Camila Crispim de Oliveira Ramos ${ }^{4}$, Fernando Campanha Bechara ${ }^{5}$
}

1 Programa de pós-graduação em Ecologia de Ambientes Aquáticos Continentais. Universidade Estadual de Maringá, Avenida Colombo, 5790, CEP 87020-900, Bloco G-90, Sala 16. Maringá, Paraná, Brasil. E-mail: <huilquer@hotmail.com>

2 Departamento de Biologia, Núcleo de Pesquisas em Limnologia, Ictiologia e Aquicultura. Universidade Estadual de Maringá, Av. Colombo 5790, Nupelia G90, Sala 18B, CEP 87020-900, Maringá, Paraná, Brasil.

3 Coordenação de Ciências Biológicas. Universidade Tecnológica Federal do Paraná. Estrada da Boa Esperança, C.P. 157, CEP 85660-000, Laboratório de Zoologia, Dois Vizinhos, Paraná, Brasil.

4 Programa de pós-graduação em Ecologia de Ambientes Aquáticos Continentais. Universidade Estadual de Maringá, Avenida Colombo, 5790, CEP 87020-900, Bloco G-90, Sala 16. Maringá, Paraná, Brasil.

5 Coordenação de Engenharia Florestal. Universidade Tecnológica Federal do Paraná. Estrada da Boa Esperança, C.P. 157, CEP 85660-000, Laboratório de Ecologia e Botânica, Dois Vizinhos, Paraná, Brasil.

Data de recebimento: 10/04/2012

Data da aprovação: 20/05/2012

\begin{abstract}
RESUMO
O estresse intensivo das variações ambientais sobre a fauna, em teoria, pode ser avaliado através de uma análise de assimetria flutuante. Deste modo, neste trabalho foi investigada a possibilidade de ocorrência de estresse ambiental através da análise de assimetria flutuante de espécies do gênero Turdus ocorrentes em um parque urbano situado em Guarapuava, Centro-Sul do Estado do Paraná, Brasil. As aves foram capturadas e tiveram asas e tarsos medidos através de paquímetro digital. Em seguida, foi obtido o índice de assimetria flutuante, obtendo-se a normalidade (simetria na distribuição dos escores). Os resultados obtidos a partir da análise de assimetria flutuante (AF) demonstraram um padrão assimétrico de tarso e asa para as três espécies em que houve número suficiente de indivíduos para análise (Turdus amaurochalinus, Turdus leucomelas e Turdus rufiventris). Embora não haja dados de ambientes mais conservados, foi detectado que ocorre assimetria flutuante para os turdídeos estudados. Desta forma, recomendam-se novos estudos com turdídeos em ambientes mais íntegros, para validar o uso desta ferramenta também sobre espécies mais resistentes ao estresse ambiental, como também para planos de manejo de unidades de conservação inseridas em matrizes urbanas.
\end{abstract}

Palavras-chave: Turdus. Habitats urbanos. Estresse ambiental. 


\begin{abstract}
The intense stress of environmental changes in wildlife can be assessed, in theory, by means of an analysis of fluctuating asymmetry. In this context, the present study aimed to investigate the possibility of environmental stress through the analysis of fluctuating asymmetry of species of Turdus in an urban park located in the municipality of Guarapuava in the mid-south region of the state of Paraná, Brazil. Birds were captured and their wings and tarsi were measured with a digital caliper. Then, the index of fluctuating asymmetry was obtained and normality was observed (symmetry in the distribution of scores). The results obtained from the analysis of fluctuating asymmetry (FA) show an asymmetrical pattern of tarsus and wing for the three more abundant species, (Turdus amaurochalinus, Turdus leucomelas and Turdus rufiventris). Although there is no data concerning more preserved environments, it is possible to point out fluctuating asymmetry for the evaluated species in that area. Therefore, further studies with thrushes are recommended in more preserved environments as an effort to better test the use of this tool on species more resistant to environmental stress, as well as for managing strategies of protected urban areas.
\end{abstract}

Keywords: Turdus. Urban habitats. Environmental stress.

\section{Introdução}

O isolamento de fauna em habitats urbanos pode ser considerado um fator causador de estresse ambiental em espécies animais. Isto porque em fragmentos florestais urbanos podem ocorrer processos de depressão endogâmica através do baixo fluxo gênico proporcionado pelo isolamento de um fragmento, bem como pelo aumento da competição (LAURENCE et al., 1998; COLLI et al., 2003; METZGER, 2006).

$\mathrm{O}$ estresse intensivo sobre a avifauna, em teoria, poderia ser avaliado através de uma análise de assimetria flutuante (AF). Este modelo de variação assume que os desvios da simetria (diferença entre o lado esquerdo e direito do corpo do animal) são distribuídos próximos a uma média zero (JUSTE et al., 2001; LEAMY et al., 2001). Percebe-se que estas medidas podem ser alteradas por perturbações ambientais que prejudicam o desenvolvimento de um organismo (VAN VALEN, 1962; KARK, 2001; PALMER; STROBECK, 2003).

Entre os fatores causadores de assimetria, ou seja, que geram instabilidade no desenvolvimento, destacam-se: variações no estado nutricional, temperatura, densidade da população, substâncias poluentes e fragmentação de habitat (MOLLER; SWADDLE, 1997; LENS et al., 1999). Portanto, a assimetria serve como uma medida do quanto o organismo conseguiu tamponar seu desenvolvimento contra condições estressantes (VAN VALEN, 1962).
Para Anciães e Marini (2000), espécies mais dependentes da integridade de habitat ou de um ambiente mais estável reagem de forma negativa às pressões impostas por estressores ambientais. O efeito de estressores ambientais sobre aves mais resistentes às alterações de habitat é pouco explorado. Entre as aves mais resistentes às alterações ambientais, podemos citar espécies da família Turdidae (COLLAR 2005; SIGRIST 2006). No Brasil, são encontradas 14 espécies do gênero Turdus (CBRO, 2011 mais a espécie recém descrita por O'NEILL, 2011). As aves deste grupo possuem razoável capacidade para voos longos, no entanto muitas espécies do gênero Turdus permanecem ao longo de todo ano defendendo seus territórios já estabelecidos (LUDVIG, 1994; CLEMENT, 2000). Desta maneira, podem sofrer com flutuações de recursos no ambiente, já que muitos dos habitats florestais urbanos restringem-se a pequenos remanescentes florestais isolados (METZGER, 2006).

São escassos os estudos de assimetria flutuante em ambientes urbanos, bem como estudos sobre o estresse ambiental como gerador de assimetria, até mesmo sobre espécies mais tolerantes às alterações do meio. Deste modo, o objetivo deste trabalho foi o de investigar a possibilidade de ocorrência de estresse ambiental através da análise de assimetria flutuante de espécies do gênero Turdus em um parque urbano situado em Guarapuava, Centro-Sul do Estado do Paraná. 


\section{Material e métodos Área de estudo}

O estudo foi realizado no Parque Municipal das Araucárias (PMA; latitude $\approx 25^{\circ} 21^{\prime} \mathrm{S}$ e longitude $\approx$ $\left.51^{\circ} 28^{\prime} \mathrm{W}\right)$, município de Guarapuava, mesorregião Centro-Sul do Estado do Paraná (Figura 1). A área de estudo possui aproximadamente 20 ha cobertos por floresta ombrófila mista alto-montana, com altitude em torno dos $1070 \mathrm{~m}$. O PMA encontra-se delimitado na parte norte pelo Rio Xarquinho, onde uma zona de formação florestal em estágio inicial de vegetação secundária faz transição com uma área de matriz agrícola. Ao sul e leste existem áreas antropizadas, e nas suas adjacências existe uma matriz urbana (CORDEIRO; RODRIGUES, 2007). O clima da região é subtropical (mesotérmico úmido), com temperatura média anual de $16,8^{\circ} \mathrm{C}$ e um regime pluviométrico próximo de $1800 \mathrm{~mm}$ anuais, caracterizando-se como tipo $C f b$ segundo a classificação de Köppen-Geiger (TOMAZ; VESTENA, 2003).
$35 \mathrm{~mm}$ ) de $6 \times 3 \mathrm{~m}$, dispostas aleatoriamente na área de estudo a uma altura de 50 a $150 \mathrm{~cm}$ do solo. As redes foram abertas das 7 às $19 \mathrm{~h}$, em quatro capturas mensais, totalizando $3.456 \mathrm{~h} / \mathrm{rede} / \mathrm{ano}$, em um esforço amostral calculado a partir de Straube e Bianconi (2002) de 7488 h.m².

Os indivíduos capturados foram classificados como adultos, filhotes ou juvenis, de acordo com a textura do tarso, dureza das unhas, plumagem e calcificação cranial (CEMAVE, 2008). Aves em processo de muda de penas foram desconsideradas para as análises durante aquela captura. Foram aferidas as seguintes variáveis biométricas em milímetros: $i$ ) massa corpórea; ii) comprimento total; iii) comprimento do bico; $i v)$ tarso direito e esquerdo; $v$ ) asa direita e esquerda; e vi) cauda. Tais dados foram obtidos através de paquímetro digital e balança analógica. Os parâmetros unilaterais mensurados foram padronizados do lado direito das aves, evitando efeitos de assimetria, de modo que as medidas foram realizadas com base em Sick (1997) e Auricchio (2001).
Figura 1 - Mapa da área de estudo (Parque Municipal das Araucárias, município de Guarapuava, Estado do Paraná, Brasil), demonstrando o fragmento florestal estudado e habitats adjacentes. Obtido de Vogel et al. (2011)

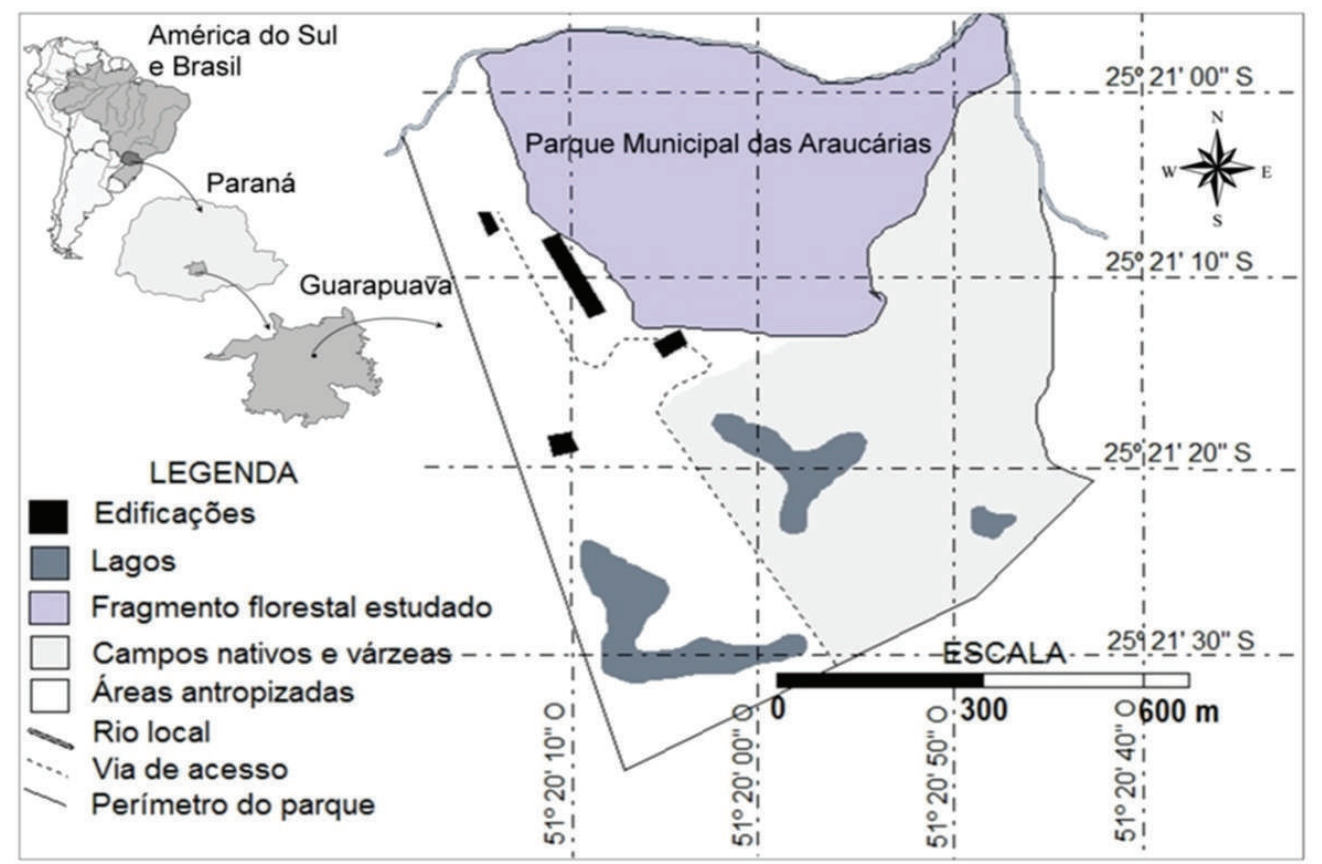

\section{Obtenção de dados}

Os dados de campo foram obtidos ao longo de 12 meses (dezembro/2008 a novembro/2009). A captura das aves foi realizada através de seis redes ornitológicas (três de malha $20 \mathrm{~mm}$ e outras três de

\section{Análise de dados}

Para a obtenção do Índice de Assimetria Flutuante (AF), foi utilizado o índice $\mathrm{AF}=[|\mathrm{D}-\mathrm{E}| /$ (D+E/2)], proposto por Palmer e Strobeck (2003), onde $\mathrm{D}=$ a medida de qualquer variável bilateral situada do lado direito (neste caso da ave), enquanto $\mathrm{E}=\mathrm{a}$ variável bilateral situado do lado esquerdo. Inicialmente, uma análise de normalidade através do teste de Shapiro-Wilk $(W)$ foi empregada sobre as três populações mais abundantes da amostra (Turdus

amaurochalinus, T. leucomelas e T. rufiventris) para as variáveis de tarso e asa. Este teste teve por objetivo averiguar os níveis de assimetria, na tentativa de evidenciar um efeito mais profundo das pressões ambientais sobre uma única espécie. Após averiguar de que modo cada população respondeu aos níveis de 
AF, o teste de Shapiro-Wilk foi aplicado considerando as cinco populações como uma única amostra, ou seja, em nível de efeito sobre a taxocenose.

\section{Resultados e discussão}

Foram capturadas cinco espécies do gênero Turdus, das quais $34 \%$ foram de T. rufiventris $(n=$ 57); em seguida, $31 \%$ de T. leucomelas ( $n=$ $51) ; 27,5 \%$ de $T$. amaurochalinus $(n=44)$; $5 \%$ de $T$. albicollis $(n=8)$; e, finalmente, $2,5 \%$ de $T$. subalaris $(n=4)$. Todavia, foi possível incluir na análise biométrica 51 indivíduos de T. rufiventris, 46 de T. leucomelas, 41 de T. amaurochalinus, oito de $T$. albicollis e quatro de T. subalaris.

Taxocenoses formadas por turdídeos são dinâmicas e variáveis. Conforme observado por Efe et al. (2007), T. rufiventris foi o turdídeo mais capturado, de modo similar aos resultados obtidos neste estudo. Gasperin e Pizo (2009) observaram que a segunda espécie mais abundante foi $T$. amaurochalinus, diferindo do presente estudo no qual a segunda mais abundante foi T. leucomelas. Aco-ocorrência de turdídeos é relativamente comum, e geralmente são citadas ocorrências de duas a quatro espécies sintópicas, como em Lopes e Anjos (2007), que confirmaram a presença de T. albicollis, T. amaurochalinus, T. leucomelas, e T. subalaris em uma área urbana no Estado do Paraná. Esta área possui características ambientais semelhantes à do presente estudo. É possível que as seis espécies do gênero Turdus ocorrentes no Estado do Paraná possam ser encontradas de forma sintópica, conforme observadas por Straube (2008) em Arapoti (noroeste do Paraná).

Os resultados obtidos a partir da análise de assimetria flutuante (AF) demonstram um padrão assimétrico de tarso e asa para as três espécies em que houve número suficiente de indivíduos para análise, com o índice de Shapiro-Wilk $(W \geq 0,70 ; P<0,05)$ para T. amaurochalinus, T. leucomelas e T. rufiventris (Tabelas 1 e 2). Efeitos assimétricos de AF também foram observados para a taxocenose em relação à variável tarso $(W=0,70 ; P<0,05)$ e asa $(W=0,86 ; P<$ 0,05 ), idem tabelas (Tabelas 1 e 2).
Considerando a abordagem sobre a ocorrência de estresse sobre turdídeos, cabe salientar que estas aves possuem hábitos alimentares onívoros (ALMEIDA et al., 2003; GASPERIN; PIZO, 2009), de modo que a onivoria atua como um tamponamento contra as flutuações no ambiente (WILLIS, 1979), amenizando os efeitos dos estressores ambientais.

\begin{tabular}{lccccc}
\hline \multicolumn{1}{c}{ Espécies } & Amostra & Média & Desvio padrão & Shapiro-Wilk & P \\
\hline T. albicollis & 8 & $4,9 \times 10^{-3}$ & $1,3 \times 10^{-3}$ & $*$ & $*$ \\
T. amaurochalinus & 41 & $1,3 \times 10^{-2}$ & $1,6 \times 10^{-3}$ & 0,880 & $<\mathbf{0 , 0 5}$ \\
T. leucomelas & 46 & $1,4 \times 10^{-2}$ & $2,6 \times 10^{-3}$ & 682 & $<\mathbf{0 , 0 5}$ \\
T. rufiventris & 51 & $1,3 \times 10^{-2}$ & $1,5 \times 10^{-3}$ & 0,882 & $<\mathbf{0 , 0 5}$ \\
T. subalaris & 4 & $3,2 \times 10^{-2}$ & $2,1 \times 10^{-2}$ & $*$ & $*$ \\
\hline Taxocenose & $\mathbf{1 5 0}$ & $\mathbf{4 , 9} \times \mathbf{1 0}^{-3}$ & $\mathbf{1 , 3} \times \mathbf{1 0}^{-2}$ & $\mathbf{0 , 7 0}$ & $<\mathbf{0 , 0 5}$ \\
\hline
\end{tabular}

Tabela 2 - Estatística descritiva referente a níveis de assimetria flutuante (AF) obtidas de asas de turdídeos ocorrentes no Parque Municipal das Araucárias, em Guarapuava, Paraná. (*) amostra insuficiente para análise

\begin{tabular}{lccccc}
\hline \multicolumn{1}{c}{ Espécies } & Amostra & Média & Desvio padrão & Shapiro-Wilk & P \\
\hline T. albicollis & 8 & $1,7 \times 10^{-2}$ & $4,7 \times 10^{-3}$ & $*$ & $*$ \\
T. amaurochalinus & 41 & $1,4 \times 10^{-2}$ & $2,3 \times 10^{-3}$ & 0,831 & $<\mathbf{0 , 0 5}$ \\
T. leucomelas & 46 & $1,5 \times 10^{-2}$ & $2,2 \times 10^{-3}$ & 0,830 & $<\mathbf{0 , 0 5}$ \\
T. rufiventris & 51 & $1,5 \times 10^{-2}$ & $1,9 \times 10^{-3}$ & 0,890 & $<\mathbf{0 , 0 5}$ \\
T. subalaris & 4 & $8,2 \times 10^{-3}$ & $2,7 \times 10^{-3}$ & $*$ & $*$ \\
\hline Taxocenose & $\mathbf{1 5 0}$ & $\mathbf{1 , 7} \times \mathbf{1 0}^{-2}$ & $\mathbf{1 , 5} \times \mathbf{1 0}^{-2}$ & $\mathbf{0 , 8 6}$ & $<\mathbf{0 , 0 5}$ \\
\hline
\end{tabular}

Desta forma, pode-se afirmar que ocorre assimetria nesta população de turdídeos. Muito embora não dispomos de informações sobre turdídeos em ambientes mais íntegros, estudos de assimetria flutuante em populações de aves em paisagens fragmentadas diagnosticam maiores níveis de assimetria nos ambientes mais alterados quando em comparação com habitats mais preservados (WAYNE et al., 1986; SARRE, 1996). Desta forma, a presença de assimetria em turdídeos evidencia a desestruturação (mesmo que parcial) das características ambientais ótimas para a manutenção destas espécies.

À medida que novos estudos em ambientes mais íntegros possam corroborar ou contrariar nossos dados, contamos com evidências indiretas do estresse 
ambiental, que podem ser constatadas, em parte, através de casos de leucismo que constantemente são observados em turdídeos que habitam parques urbanos (GONÇALVES Jr. et al., 2008). Tais fenômenos são ocasionados pela endogamia, um dos agentes causadores de assimetria (MOLLER; MOUSSAEU, 2001). Assim, dependendo da pressão que o ambiente exerce sobre as espécies de turdídeos, os efeitos podem ter maior ou menor grau de severidade.

No nível de taxocenose não é possível inferir categoricamente qual estressor deve ter maior peso sobre os organismos. No entanto, a pressão sobre a qualidade do habitat (e.g. fragmentação) exerce algum efeito, como o constatado por Anciães e Marini (2000). De acordo com Grieco (2003), padrões assimétricos de tarso ocorrem principalmente por estresse nutricional durante o crescimento, enquanto padrões assimétricos de asas estão relacionados com a ocorrência de poluentes orgânicos (JANSEN et al., 2010).

Houve um efeito assimétrico sobre as estruturas morfológicas averiguadas, evidenciando que a assimetria flutuante que é utilizada como indicador do efeito da desestruturação ou perturbação de habitats pode ser utilizada sobre espécies tolerantes às alterações em ecossistemas urbanos. Desta forma, recomendam-se novos estudos com turdídeos em ambientes mais íntegros, para validar o uso desta ferramenta também sobre espécies mais resistentes ao estresse ambiental, sendo eventualmente útil para estudos e planos de manejo de unidades de conservação inseridas em matrizes urbanas.

\section{Agradecimentos}

Agradecemos ao IBAMA (licença de pesquisa $n^{\circ}$ 160531/ CEMAVE/SNA, processo $n^{\circ} 30311$ ). Igualmente agradecemos ao MCNG e SEMAFLOR pela autorização de pesquisa no Parque Municipal das Araucárias. Agradecemos à Coordenação de Aperfeiçoamento de Pessoal de Nível Superior (CAPES) e ao $\mathrm{CNPq}$ (Conselho Nacional de Desenvolvimento Científico e Tecnológico), pela bolsa de doutorado de HFV e CCOR; e de apoio financeiro a CHZ, processo 306066/2009-2; além do Programa de Excelência Acadêmica (PROEX) da CAPES (Coordenação de Aperfeiçoamento de Pessoal de Nível Superior) pelo apoio técnico e financeiro ao Programa de Pós-graduação em Ecologia de Ambientes Aquáticos Continentais.

\section{Referências}

ALMEIDA, A.; COUTO, H. T. Z.; ALMEIDA, A. F. Diversidade beta de aves em habitats secundários da Pré-Amazônia Maranhense e interação com modelos nulos. Ararajuba, São Leopoldo, v. 11, n. 1, p. 157-171, 2003.

ANCIÃES, M.; MARINI, M. A. The effects of fragmentation on fluctuating asymmetry in passerine birds of Brazilian tropical forests. Journal of Applied Ecology, London, v. 37, n. 6 , p. 1013-1028, 2000. Disponível em: <http:// dx.doi.org/10.1046/j.1365-2664.2000.00554.x>.

AURICCHIO, P. Aves. In: AURICCHIO, P.; SALOMÃO, M. G., Eds. Técnicas de coleta e preparação de vertebrados. São Paulo: Instituto Pau Brasil História Natural, FAPESP, 2001. p.127-148.

CBRO (COMITÊ BRASILEIRO DE REGISTROS ORNITOLÓGICOS). Listas das aves do Brasil. 10.ed. Disponível em: <http://www.cbro.org.br>. Acesso em: 05 abril 2012.

CEMAVE. Manual de anilhamento de aves silvestres. Brasília: MMA, 2008.

CLEMENT, P. Thrushes. Princeton: Princeton University Press, 2000.

COLLAR, N. J. Family Turdidae (Thrushes). In: DEL HOYO, J.; ELLIOT, A.; CHRISTIE, D. A. (Eds.). Handbook of the birds of the world. Barcelona: Lynx Edicions, 2005.

COLLI, G. R.; ACCACIO, G. M.; ANTONINI, Y.; CONSTANTINO, R.; FRANCESCHINELLI, E. V.; LAPS, R. R.; SCARIOT, A. O.; VIEIRA, M. V.; WIEDERHECKER, C. A. Fragmentação dos ecossistemas e a biodiversidade brasileira: uma síntese. In: RAMBALDI, D. M.; OLIVEIRA, D. A. O. (Eds.). Fragmentação de ecossistemas: causas, efeitos sobre a biodiversidade e recomendações de políticas públicas. Brasília, Ministério do Meio Ambiente, Secretaria de Biodiversidade e Floretas, 2003. p. 317-324.

CORDEIRO, J.; RODRIGUES, W. A. Caracterização fitossociológica de um remanescente de Floresta Ombrófila mista em Guarapuava, PR. Revista Árvore, Viçosa, v. 31, n. 3, p. 545-554, 2007. Doi: http://dx.doi.org/10.1590/S010067622007000300020

EFE, M. A.; OLIVEIRA, A. C.; KOCH, M.; FLÔRES, J. M.; SCHERER, S. B. Avifauna da Área de Proteção Ambiental do Ibirapuitã, Rio Grande do Sul, Brasil. Ornithologia, Palmas, v. 2, n. 1, p. 14-24, 2007.

GASPERIN, G.; PIZO, M. A. Frugivory and habitat use by thrushes (Turdus spp.) in a suburban area in south Brazil. Urban Ecosystems, Duluth, v. 12, n. 1, p. 425-436, 2009. Doi: http://dx.doi.org/10.1007/s11252-009-0090-2

GONÇALVES JR., C. C.; SILVA, E. A.; DE LUCA, A. C.; PONGILUPPI, T.; MOLINA, F. B. Record of a leucistic 
Rufous-bellied Thrush Turdus rufiventris (Passeriformes, Turdidae) in São Paulo city, Southeastern Brazil. Revista Brasileira de Ornitologia, São Paulo, v. 16, n. 1, p. 72-75, 2008.

GRIECO, F. Greater food availability reduces tarsus asymmetry in nestling blue tits. The Condor, Los Angeles, v. 105, n. 3, p. 599-603, 2003. Doi: http://dx.doi. org $/ 10.1650 / 7177$

JANSEN, B. M.; AARNES, J. B.; MURVOLL, K. M.; HERZKE, D.; NYGARD, T. Fluctuating wing asymmetry and hepatic concentrations of persistent organic pollutants are associated in European shag (Phalacrocorax aristotelis) chicks. Science of the Total Environment, Bethesda, v. 408, n. 3, p. 578-585, 2010. Doi: http://dx.doi.org/10.1016/j. scitotenv.2009.10.036

JUSTE, J. C.; LÓPEZ, G.; STRAUSS, R. E. Analysis of asymmetries in the african fruit bats Eidolon helvum and Rousettus egyptiacus (Mammalia: Megachiroptera) from the islands of the gulf of Guinea. II. Integration and levels of multivariate fluctuating asymmetry across a geographical range. Journal of Evolutionary Biology, Basle, v. 14, n. 4, p. 672-680, 2001. Doi: http://dx.doi.org/10.1046/j.14209101.2001.00299.x

KARK, S. Shifts in bilateral asymmetry within a distribution range: The case of the Chukar partridge. Evolution, Washington v. 55, n. 10, p. 2088-2096, 2001. Doi: http:// dx.doi.org/10.1111/j.0014-3820.2001.tb01323.x

LAURANCE, S. G.; HUTCHINGS, R. W.; LOVEJOY, T. E. Effects of forest fragmentation on recruitment patterns in Amazonian tree communities. Conservation Biology, Gainesville, v. 12, n. 2, p. 460-464, 1998. Doi: http://dx.doi. org/10.1111/j.1523-1739.1998.97175.x

LEAMY, L.; MEAGHER, J.; TAYLOR, S.; CARROLL, L.; POTTS, W. K. Size and fluctuating asymmetry of morphometric characters in mice: their associations with inbreeding and t-haplotype. Evolution, Washington, v. 55, n. 11, p. 2333-2341, 2001. Doi: http://dx.doi.org/10.1554/ 0014-3820(2001)055[2333:SAFAOM]2.0.CO;2

LENS, L.; VAN DONGEN, S.; WILDER, C. M.; BROOKS, T. M.; MATTHYSEN, E. Fluctuating asymmetry increases with habitat disturbance in seven bird species of a fragmented afrotropical forest. Procedings of The Royal Society, Bethesda, v. 266, n. 22, 1241-1246, 1999. Doi: http://dx.doi.org/10.1098/rspb.1999.0769

LOPES, E. V.; ANJOS, L. A composição da avifauna da Universidade Estadual de Londrina, Norte do Paraná, Brasil. Revista Brasileira de Zoologia, Curitiba, v. 23, n. 1, p. 145-156, 2007. Doi: http://dx.doi.org/10.1590/ S0101-81752006000100005

LUDVIG, E.; TÖRÖK, J.; VANICSEK, L.; CSÖRGÖ, T. Territoriality and population regulation in urban Blackbirds (Turdus merula L.). Ornis Hungarica, Budapest v. 4, n.1, p. 1-8. 1994.
METZGER, J. P. Como lidar com regras pouco óbvias para conservação da biodiversidade em paisagens fragmentadas. Natureza \& Conservação, Curitiba, v. 4, n. 2, p. 11-23, 2006 .

MØLLER, A. P.; SWADDLE, B. Developmental stability and evolution. Oxford, Oxford University Press, 1997. 291p.

.; MOUSSEAU, T. A. Albinism and phenotype of barn swallows (Hirundo rustica) from Chernobyl. Evolution, Washington, v. 55, n. 10, p. 2097-2104, 2001. Doi:54/0014-3820(2001)055[2097:AAPOBS]2.0.CO;2

O'NEILL, J. O.; LANE, D. F.; NAKA, L. N. A cryptic new species of thrush (Turdidae: Turdus) from western amazonia. The Condor, Berkeley, v. 113, n. 4, p. 869-880, 2011. Doi: http://dx.doi/10.1525/cond.2011.100244

PALMER, A. R.; STROBECK, C. Fluctuating asymmetry analyses revisited. In: POLAK, M. (Ed.). Developmental instability: causes and consequences. Oxford, Oxford University Press, 2003. p. 279-319.

SARRE, S. Habitat fragmentation promotes fluctuating asymmetry but not morphological divergence in two geckos. Researches on Population Ecology, Kyoto v. 38, n. 1, p. 57-6, 1996. Doi: http://dx.doi.org/10.1007/BF02514971

SICK, H. Ornitologia brasileira. Rio de Janeiro, Nova Fronteira, 1997, 862p.

SIGRIST, T. Aves do Brasil: uma visão artística. São Paulo: Fosfertil, 2006.

STRAUBE, F. C. Avifauna da Fazenda Barra Mansa (Arapoti, Paraná) com anotações sobre a ocupação de monoculturas de essências arbóreas. Atualidades Ornitológicas, Ivaiporã, v. 142, n. 1, p. 46-50, 2008.

STRAUBE, F. C.; BIANCONI, G. V. Sobre a grandeza e a unidade utilizada para estimar o esforço de captura com a utilização de redes - de - neblina. Chiroptera Neotropical, Brasília, v. 8, n. 2, p.150-152, 2002.

THOMAZ, E. L.; VESTENA, L. R. Aspectos climáticos de Guarapuava-PR. Guarapuava, Editora da Unicentro, 2003.

VAN-VALEN, L. A study of fluctuating asymmetry. Evolution, Washington, v. 16, p. 125-142, 1962.

VOGEL, H. F.; ZAWADZKI, C. H.; METRI, R. Coexistência entre Turdus leucomelas Vieillot, 1818 e Turdus rufiventris Vieillot, 1818 (Aves: Passeriformes) em um fragmento urbano de floresta com araucárias, Sul do Brasil. Biota Neotropica, Campinas, v. 11, n. 3, p.35-45, 2011. Doi: http://dx.doi.org/10.1590/S1676-06032011000300002.

WAYNE, R. K., MODI, W. S.; O'BRIEN. S. J. Morphological variability and asymmetry in the Cheetah (Acinonyx jubatus), genetically uniform species. Evolution, Washington, v. 40, n. 1, p. 78-85, 1986.

WILLIS, E. O. The composition of avian communities in remanescent woodlots in southern Brazil. Papéis Avulsos de Zoologia, São Paulo, v. 3, n. 1, p. 1-25, 1979. 